\title{
Association of Sleep Duration and Working Hours with Suicidal Ideation in Shift Workers: The Korean National Health and Nutrition Examination Survey 2007-2018
}

\author{
Sun-Young Kim², Soo In Kim², and Weon-Jeong Lim ${ }^{1}$ \\ 1'Department of Psychiatry, Ewha Womans University Seoul Hospital, Ewha Womans University College of Medicine, Seoul, Republic of Korea \\ ${ }^{2}$ Department of Psychiatry, Ewha Womans University Mokdong Hospital, Ewha Womans University College of Medicine, Seoul, Republic of Korea
}

\begin{abstract}
Objective This study investigated whether sleep duration and working hours were associated with the risk of suicidal ideation.
Methods Data from 13,628 shift workers (age $\geq 19$ ) were obtained from the nationwide cross-sectional Korea National Health and $\mathrm{Nu}$ trition Examination Surveys conducted in 2007-2018. We included healthy shift workers without depressive disorders and chronic medical illnesses. Sleep duration, working hours, and suicidal ideation were assessed using a self-reported questionnaire. Logistic regressions were used to examine the association of sleep duration and working hours with the risk of suicidal ideation. We examined interactions between sleep duration and working hours in association with suicidal ideation. In addition, interactions of sex or age were also analyzed.

Results Shift workers sleeping for $<6$ and $\geq 10$ hours/day were associated with suicidal ideation compared with those sleeping for 7 to $<9$ hours/day. Individuals working $>52$ hours/week had a higher risk of suicidal ideation compared with those working $\leq 40$ hours/week. In terms of interaction by sex or age groups in the association between working hours and the risk of suicidal ideation, the relationship was stronger for men than for women and for those aged $<45$ years than for those aged $\geq 45$ years.

Conclusion Shorter or longer sleep durations, and long working hours were associated with a higher risk of suicidal ideation. Under long working hours, male shift workers or those aged $<45$ years were more vulnerable to suicidal ideation.
\end{abstract}

Psychiatry Investig 2021;18(5):400-407

Key Words Shift work, Working hours, Sleep duration, Suicidal ideation.

\section{INTRODUCTION}

Suicide is a global public health crisis. Close to 800,000 people die due to suicide every year worldwide. ${ }^{1}$ The suicide rate in South Korea is 24.6 per 100,000 people, which is the highest of any nation in the Organisation for Economic Co-operation and Development. ${ }^{2}$ Prevention is the only way to reduce suicide; therefore, it is important to identify and reduce modifiable risk factors for suicide. ${ }^{3}$ Previous studies on suicide in occupational settings have identified certain risk factors and suggested interventions.

Received: November 26, 2020 Accepted: February 1, 2021

$\square$ Correspondence: Weon-Jeong Lim, MD, PhD

Department of Psychiatry, Ewha Womans University Seoul Hospital, Ewha Womans University College of Medicine, 260 Gonghang-daero, Gangseo-gu, Seoul 07804, Republic of Korea

Tel: +82-2-6986-1676, Fax: +82-2-6986-3066, E-mail: psyweon@ewha.ac.kr

(c) This is an Open Access article distributed under the terms of the Creative Commons Attribution Non-Commercial License (https://creativecommons.org/licenses/bync/4.0) which permits unrestricted non-commercial use, distribution, and reproduction in any medium, provided the original work is properly cited.
In modern society, people who work non-day time hours are required to manage the demands for 24-hour consumer service. Approximately $15-30 \%$ of employee are engaged in a shift-work system, ${ }^{4,5}$ with some indication that the prevalence of shift work is increasing. ${ }^{6}$ However, shift work disrupts circadian rhythms and interferes with social obligations and/or family life that occur during the day. This leads to increased vulnerability to the development of stress-related mental health problems, including suicide. ${ }^{7}$

Work hours that result in non-standard sleep can impair an individual's ability to sleep and cause shorter sleep durations, severe sleep debt, and daytime sleepiness with a more prolonged recovery time. ${ }^{8}$ A recent review has reported that shift workers have shorter total sleep times than daytime workers. ${ }^{9}$ In contrast, Härmä et al. ${ }^{10}$ have suggested that shift work with/without night shifts are associated with increased fatigue and longer sleep times ( $\geq 9$ hours/day). Sleep disturbances related with shift work are a central mechanism for the relationship between shift work and suicide; ${ }^{7}$ therefore, it is important to investigate 
the dose-response relationship between sleep duration and suicidal ideation and find the appropriate sleep duration that reduces the risk of suicidal ideation.

In addition, working hours should be also considered as a factor to think together with sleep because long working hours counteract sufficient sleep time in a shift work system. Lee et al. ${ }^{11}$ have reported that long working hours ( $>45$ hours/week) are associated with suicidal mortality in general workers. They argued that long working hours increases the risk of poor mental health, social isolation, and family conflict. Given shift work interferes with appropriate sleep and social activities that occur during the day, ${ }^{7}$ shift workers with long working hours can be more vulnerable to the risk of suicide than non-shift workers. However, few studies have investigated the appropriate length of work time for shift workers to reduce this risk.

The aim of this study was to use a nationally representative survey to address three questions. First, are work hours and sleep duration associated with the risk of suicidal ideation in shift workers? Second, what is the appropriate length of work hours and sleep duration to reduce the risk of suicidal ideation? Third, is there an interaction between work hours and sleep duration in their associations with the risk of suicidal ideation?

\section{METHODS}

\section{Participants}

This study used data collected from the Korean National Health and Nutrition Examination Survey (KNHANES), which is a nationally representative, cross-sectional survey that has been conducted annually by the Korea Centers for Disease Control and Prevention (KCDC) since 1998. ${ }^{12}$ The KNHANES consists of health interviews, health examinations, and dietary surveys to evaluate the health and nutritional status of the Korean population. Participants of the KNHANES are selected using a complex, stratified, multi-stage cluster sampling design among non-institutionalized civilians in South Korea. ${ }^{12}$ Self-reported sociodemographic characteristics, such as age, sex, marital status, education level, income, alcohol consumption, smoking status, sleep duration, quality of life, and personal medical history, are collected during the health interview. Anthropometric and blood biochemical data are measured during the health examination. All participants provide written informed consent, and the survey protocol was approved by the KCDC Institutional Review Board (IRB Nos. 2007-02CON-04-P, 200804EXP-01-C, 2009-01CON-03-2C, 2010-02CON-21-C, 201102CON-06-C, 2012-01EXP-01-2C, 2013-07OCN-03-4C, 201312EXP-03-5C, 2015-01-02-6C, 2018-01-03-P-A) (KCDC, 2020).

\section{Selection of "healthy" shift workers}

The current study used a cross-sectional design of 58,223 work- ers aged 19 and older who had participated in the KNHANES between January 2007 and December 2018. The definition of shift work by Knutsson ${ }^{13}$ is "an arrangement of working hours that uses teams (shifts) of workers, in order to extend the hours of operation of the work environment beyond that of the conventional office hours." Therefore, individuals who reported being employed with fixed standard time schedules (06:0018:00) were excluded $(n=32,130)$. We selected "healthy" shift workers without any evidence of a depressive disorder $(n=1,829)$ or physical illness (cerebral infarction, myocardial infarction/ angina, chronic renal failure, liver cirrhosis, and cancer; $n=1,488$ ) to reduce reverse causation. We excluded participants with missing data $(n=15,990)$. Finally, 13,628 participants were included in our analysis (Figure 1).

\section{Assessment of sleep duration}

Participants were asked, "How many hours do you usually sleep a day?" We categorized the responses into five groups: $<6,6$ to $<7,7$ to $<9,9$ to $<10, \geq 10$ hours/day. Based on a joint consensus statement of the American Academy of Sleep Medicine and Sleep Research Society, ${ }^{14}$ we defined individuals sleeping between 7 and $<9$ hours/day as a reference group.

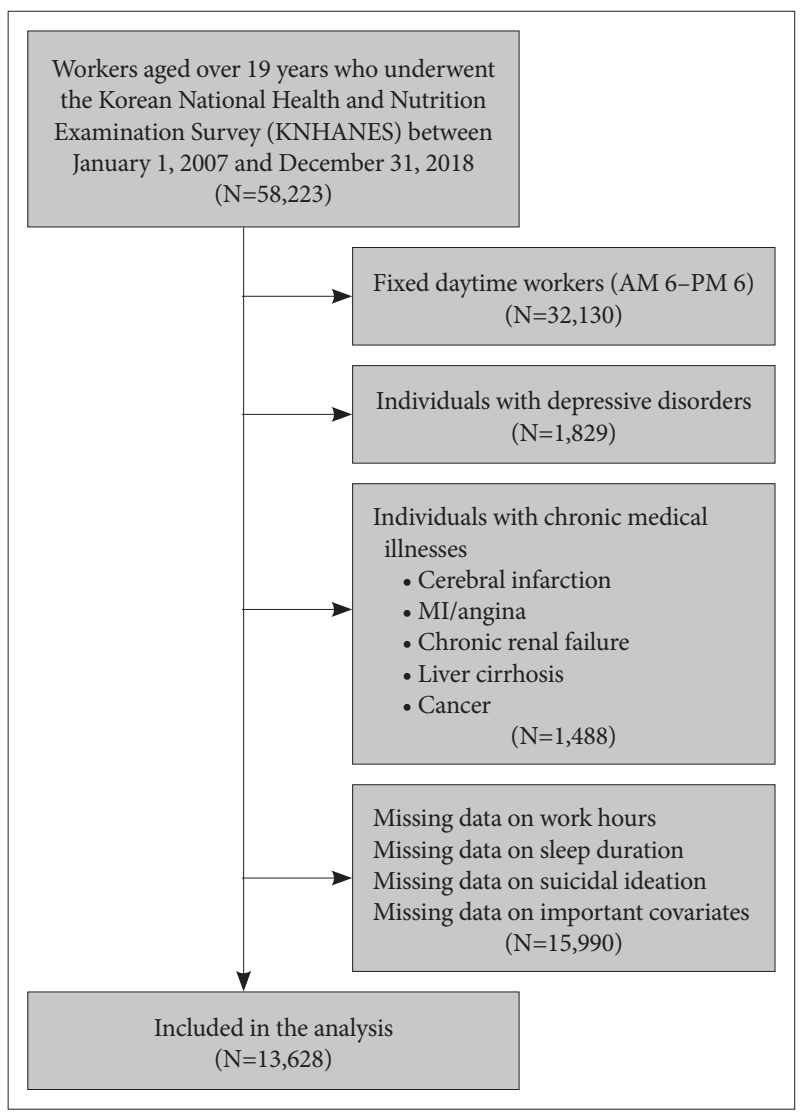

Figure 1. Overview of the study participant selection. 


\section{Assessment of working hours}

Participants were asked, "How many hours do you usually work per week, including overtime?” The Labor Standard Act in Korea defines standard working hours as 40 hours/week, with extensions up to 52 hours permitted with the worker's consent. ${ }^{15}$ Based on this Act, working hours were divided into three groups: $\leq 40,40.1-52$, and $>52$ hours/week.

\section{Assessment of suicidal ideation}

Participants were asked, "Over the last year, have you ever felt that you would be better off dead?" Answers in the affirmative were taken to indicate suicidal ideation.

\section{Potential confounding variables}

Age, sex, marital status (married, never married, and others), education (less than elementary school, middle school, high school diploma, and college degree or higher), income (1st, 2nd, 3rd, and 4th quartiles), alcohol consumption frequency ( $\leq 1$ time/month, 2-4 times/month, 2-3 times/week, and $\geq 4$ times/week), and smoking status (never smoker, former smoker, and current smoker) were collected using the self-reported questionnaires. The level of anxiety and depressive symptoms was measured using the anxiety/depression items on the Euro-QoL-5D. ${ }^{16}$ We excluded individuals with depressive disorders; however, some clinically depressed individuals may not have been diagnosed at the time of survey. Therefore, anxiety/ depressive symptoms were included as a covariate. BMI was calculated by dividing weight $(\mathrm{kg})$ by height squared $\left(\mathrm{m}^{2}\right)$.

We collected data regarding shift work patterns, occupations, and employment status. Shift workers are divided in KNHANES as follows: "fixed-evening shift (14:00-24:00), fixed-night shift (21:00-08:00), regular day and night rotating shift, 24-hours rotating shift, split shift (working 2 shifts in 1 day), irregular rotating shift, and others. We used ten categories of workers based on the International Standard Classification of Occupations of Internal Labor Organization: ${ }^{17}$ 'managers', 'professionals,' 'office workers,' 'service workers,' 'sales workers,' 'agricultural, forestry and fishery workers,' 'craft and related trades workers,' 'plant and machine operators and assemblers', 'elementary occupations,' 'armed forces', and 'others.' Employment status was divided into four groups: 'wage workers,' 'owneroperators and employers,' 'unpaid family worker,' and 'others.'

\section{Statistical analysis}

Descriptive statistics were used to display the baseline characteristics according to suicidal ideation. The t-test and chisquare test were used to identify differences between individuals with or without suicidal ideation. Logistic regression was used to calculate the odds ratios (ORs) for the risk of suicidal ideation according to sleep duration or working hours. The rel- ative confounding effect of each of the variables outlined above was investigated in turn, before a final multivariate model including all covariates was constructed. Regression models were mutually adjusted for sleep duration and working hours to examine whether they affected each other's relationship with the risk of suicidal ideation.

Interactions between sleep duration and working hours were also examined. In addition, considering sex and age difference, interactions by sex and age were analyzed. All statistical analyses were performed using STATA version 14.0 (StataCorp LLC., College Station, TX, USA). A two-tailed p-value $<0.05$ was considered significant.

\section{RESULTS}

Table 1 displays the characteristics of the participants according to their risk of suicidal ideation. We found that 1,443 out 13,628 (10.59\%) had suicidal ideation. The groups that expressed suicidal ideation were more likely to be older, women, never smokers, have lower education and BMI, higher income levels, alcohol consumption frequency, and anxiety/depressed symptoms when compared with those without suicidal ideation. In addition, the suicidal ideation group was more likely to report shorter sleep durations than the non-suicidal ideation group.

Table 2 displays the occupational characteristics of the participants according to their risk of suicidal ideation. Participants without suicidal ideation were more likely to have fixed-evening, fixed-night, regular day and night rotating, 24-hours rotating, split, or irregular rotating shifts when compared with those without suicidal ideation. The groups that had suicidal ideation were more likely to be sales workers, agricultural, forestry and fishery workers, elementary occupations, owneroperators and employers, and unpaid family workers when compared with those with no reported suicidal ideation. In addition, the suicidal ideation group was more likely to report longer working hours than the non-suicidal ideation group.

Table 3 shows the results for the relationship between sleep duration and risk of suicidal ideation. There was little difference in the OR for suicidal ideation before versus after adjusting for sleep duration (models 3 vs. 4). In the final model, which was adjusted for confounders including sociodemographic factors, occupation characteristics, anxiety/depressive symptoms, and sleep duration, shift workers who slept $<6$ and $\geq 10$ hours/day had a $36 \%(95 \% \mathrm{CI}=15-60 \%, \mathrm{p}<0.001)$ and $42 \%(95 \% \mathrm{CI}=3-$ $95 \%, \mathrm{p}=0.031$ ) increased risk of suicidal ideation, respectively, compared with those who slept 7 to $<9$ hours/day (reference group). There was no significant association between sleeping 6 to $<7$ or 9 to $<10$ hours/day and the risk of suicidal ideation compared with the reference group. There was a negative relationship between continuous sleep duration and the risk of 
Table 1. Sociodemographic characteristics of the participants according to suicidal ideation

\begin{tabular}{|c|c|c|c|}
\hline & Without suicidal ideation $(\mathrm{N}=12,185)$ & With suicidal ideation $(\mathrm{N}=1,443)$ & \\
\hline & Mean \pm SD or $N(\%)$ & Mean \pm SD or $\mathrm{N}(\%)$ & \\
\hline Age & $45.34 \pm 0.13$ & $48.13 \pm 0.41$ & $<0.001$ \\
\hline \multicolumn{4}{|l|}{ Sex } \\
\hline Men & $6,817(56.00)$ & $564(39.09)$ & \multirow{2}{*}{$<0.001$} \\
\hline Women & $5,368(44.05)$ & $879(60.91)$ & \\
\hline \multicolumn{4}{|l|}{ Marital status } \\
\hline Married & $9,949(81.65)$ & $1,201(83.23)$ & \multirow{3}{*}{0.314} \\
\hline Never married & $2,222(18.24)$ & $241(16.70)$ & \\
\hline Other & $14(0.11)$ & $1(0.07)$ & \\
\hline \multicolumn{4}{|l|}{ Education } \\
\hline Less than elementary school & $2,050(16.82)$ & $483(33.47)$ & \multirow{4}{*}{$<0.001$} \\
\hline Middle school & $1,331(10.92)$ & $166(11.50)$ & \\
\hline High school diploma & $4,661(38.25)$ & $471(32.64)$ & \\
\hline College degree or higher & $4,143(34.00)$ & $323(22.38)$ & \\
\hline \multicolumn{4}{|l|}{ Income } \\
\hline 1st quartile & $2,638(21.65)$ & $443(30.70)$ & \multirow{4}{*}{$<0.001$} \\
\hline 2nd quartile & $3,174(26.05)$ & $377(26.13)$ & \\
\hline 3rd quartile & $3,235(26.55)$ & $367(25.43)$ & \\
\hline 4th quartile & $3,138(25.75)$ & $256(17.74)$ & \\
\hline \multicolumn{4}{|l|}{ Alcohol consumption frequency } \\
\hline$\leq 1$ time/month & $3,357(27.55)$ & $434(30.08)$ & \multirow{5}{*}{$<0.001$} \\
\hline 2-4 times/month & $4,440(36.44)$ & $447(30.98)$ & \\
\hline 2-3 times/week & $2,309(18.95)$ & $211(14.62)$ & \\
\hline$\geq 4$ times/week & $1,033(8.48)$ & $176(12.20)$ & \\
\hline Other & $1,046(8.58)$ & $175(12.13)$ & \\
\hline \multicolumn{4}{|l|}{ Smoking status } \\
\hline Never smoker & $6,085(49.94)$ & $820(56.83)$ & \multirow{3}{*}{$<0.001$} \\
\hline Former smoker & $1,066(8.75)$ & $41(2.84)$ & \\
\hline Current smoker & $5,034(41.31)$ & $582(40.33)$ & \\
\hline \multicolumn{4}{|l|}{ Anxiety/depressive symptoms (EQ-5D) } \\
\hline Not anxious or depressed & $11,394(93.51)$ & $1,000(69.30)$ & \multirow{3}{*}{$<0.001$} \\
\hline Moderately anxious or depressed & $764(6.27)$ & $411(28.48)$ & \\
\hline Extremely anxious or depressed & $27(0.22)$ & $32(2.22)$ & \\
\hline BMI & $23.76 \pm 0.03$ & $23.45 \pm 0.10$ & 0.001 \\
\hline Sleep duration & $6.84 \pm 0.01$ & $6.65 \pm 0.04$ & $<0.001$ \\
\hline
\end{tabular}

BMI: body mass index, EQ-5D: Euro-QoL-5D

suicidal ideation; however, the odds ratio was almost $1(\mathrm{OR}=$ 0.93, 95\% CI=0.89-0.97, $\mathrm{p}=0.001$ ).

Table 4 shows the relationship between working hours and the risk of suicidal ideation. There was little difference in the OR for suicidal ideation before versus after adjusting for working hours (model 3 vs. model 4). In the final model, which was adjusted for a range of confounders including sociodemographic factors, occupation characteristics, anxiety/depressive symptoms, and working hours, shift workers who worked $>52$ hours/week had a 30\% (95\% CI=13-49\%, p<0.001) increased risk of suicidal ideation compared with those who worked $\leq 40$ hours/week (reference group). There was no significant association between working 40.1-52 hours/week and the risk of suicidal ideation in shift workers when compared with the reference group. There was a positive relationship between continuous working hours and the risk of suicidal ideation; how- 
Table 2. Occupational characteristics of the participants according to suicidal ideation

\begin{tabular}{|c|c|c|c|}
\hline & Without suicidal ideation $(\mathrm{N}=12,185)$ & With suicidal ideation $(\mathrm{N}=1,443)$ & \\
\hline & Mean \pm SD or $\mathrm{N}(\%)$ & Mean \pm SD or $\mathrm{N}(\%)$ & \\
\hline \multicolumn{4}{|l|}{ Shift work patterns } \\
\hline Fixed-evening shift & $2,714(22.27)$ & $180(12.47)$ & \multirow{7}{*}{$<0.001$} \\
\hline Fixed-night shift & $746(6.12)$ & $71(4.92)$ & \\
\hline Regular day and night rotating shift & $953(7.82)$ & $40(2.77)$ & \\
\hline 24-hours rotating shift & $438(3.59)$ & $30(2.08)$ & \\
\hline Split shift & $310(2.54)$ & $24(1.66)$ & \\
\hline Irregular rotating shift & $352(2.89)$ & $35(2.43)$ & \\
\hline Others & $6,672(54.76)$ & $1,063(73.67)$ & \\
\hline \multicolumn{4}{|l|}{ Occupation } \\
\hline Managers & $352(2.89)$ & $33(2.29)$ & \multirow{11}{*}{$<0.001$} \\
\hline Professionals & $2,254(18.50)$ & $186(12.89)$ & \\
\hline Office workers & $1,312(10.77)$ & $127(8.80)$ & \\
\hline Service workers & $1,594(13.08)$ & $159(11.02)$ & \\
\hline Sales workers & $1,465(12.02)$ & $180(12.47)$ & \\
\hline Agricultural, forestry \& fishery workers & $1,262(10.36)$ & $261(18.09)$ & \\
\hline Craft and related trades workers & $903(7.41)$ & $107(7.42)$ & \\
\hline Plant and machine operators and assemblers & $1,182(9.70)$ & $98(6.79)$ & \\
\hline Elementary occupations & $1,813(14.88)$ & $285(19.75)$ & \\
\hline Armed forces & $40(0.33)$ & $3(0.21)$ & \\
\hline Others & $8(0.07)$ & $4(0.28)$ & \\
\hline \multicolumn{4}{|l|}{ Employment status } \\
\hline Wage workers & $7,668(62.93)$ & $804(55.72)$ & \multirow{4}{*}{$<0.001$} \\
\hline Owner-operators \& employers & $3,772(30.96)$ & $495(34.30)$ & \\
\hline Unpaid family worker & $734(6.02)$ & $142(9.84)$ & \\
\hline Others & $11(0.09)$ & $2(0.14)$ & \\
\hline Average work time & $44.75 \pm 0.18$ & $46.65 \pm 0.55$ & 0.001 \\
\hline
\end{tabular}

ever, the odds ratio was almost $1(\mathrm{OR}=1.006,95 \% \mathrm{CI}=1.003-$ $1.009, \mathrm{p}<0.001)$.

There was no evidence of an interaction between sleep duration and working hours with the risk of suicidal ideation $(p=0.931)$. There were interactions by sex or when stratified by age group (greater than or less than 45 years old) in the association between long working hours and the risk of suicidal ideation; the relationship was stronger for men than for women $(\mathrm{p}<0.01)$ and for those aged $<45$ years than for those aged $\geq 45$ years $(\mathrm{p}<0.01)$. However, there were no interactions by sex $(p=0.459)$ or age $(p=0.303)$ in the association between sleep duration and suicidal ideation.

\section{DISCUSSION}

The results of this study showed that both too little ( $<6$ hours/ day) and too much ( $\geq 10$ hours/day) sleep and working $>52$ hours/week were associated with the risk of suicidal ideation.

Previous studies have reported that both short and long sleep durations are associated with the risk of suicide. ${ }^{18,19}$ Littlewood et al. ${ }^{20}$ have reported that restoring sleep duration can offer temporary relief from waking problems; therefore, they argue that short sleep durations may counteract helpful interventions that are used before establishing a permanent resolution of suicidal ideation. Conversely, in terms of the relationship between long sleep duration and suicide, cytokines, such as IL-6, can function as mediators between long sleep duration and suicide. IL- 6 is somnogenic and associated with a pro-inflammatory state that contributes to a higher suicide risk. ${ }^{18}$ In addition, the overuse of opioid and sedative/hypnotic drugs that result in longer sleep may mediate the association with increasing suicidal ideation; participants who overuse opioid and sedative/hypnotic drugs are more likely to report psychiatric difficulties and chronic pain related to suicide. ${ }^{21}$ 

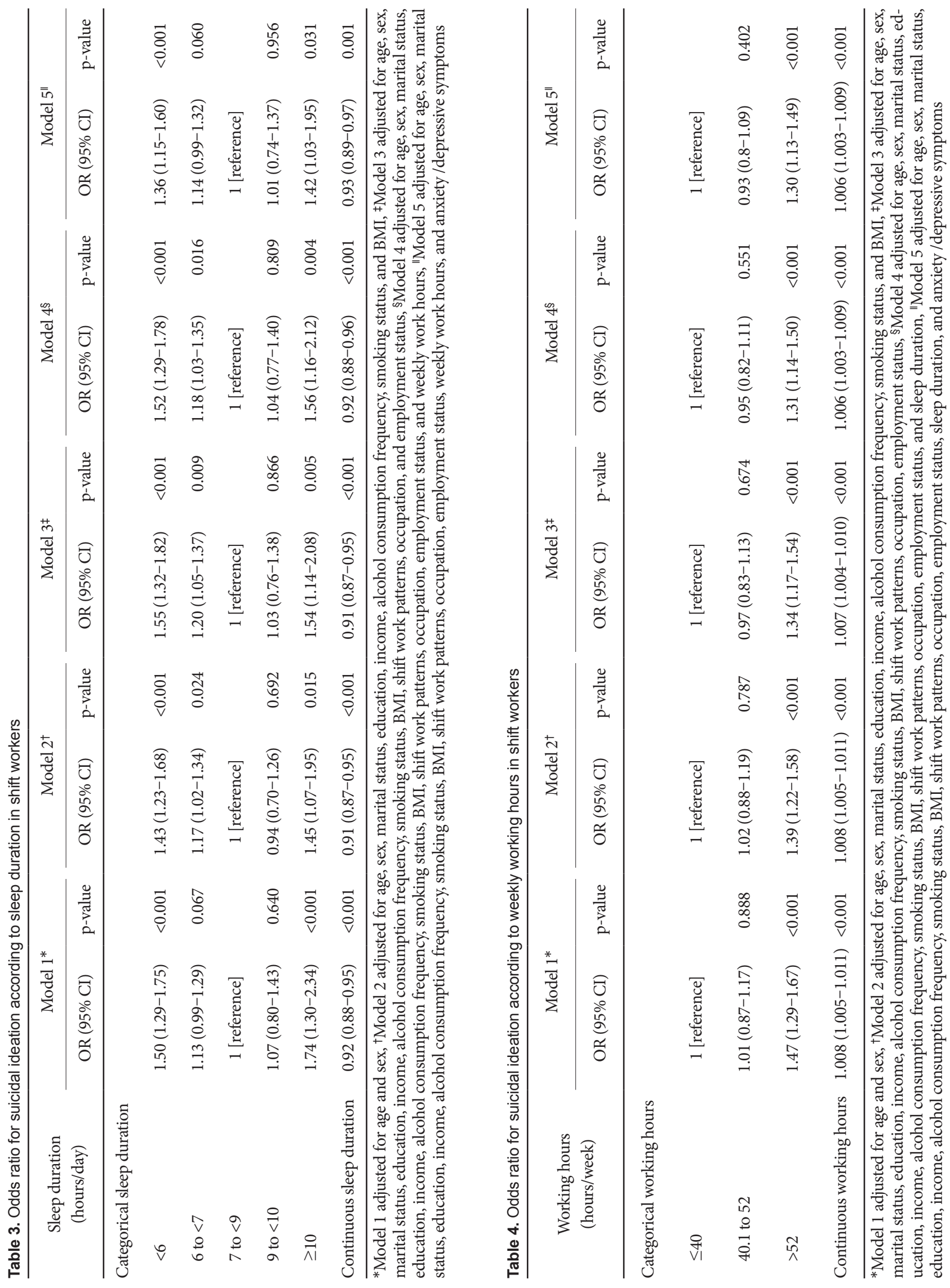
Shift work causes a desynchronization between homeostatic sleep pressure and circadian rhythmicity. ${ }^{22}$ Shift workers can sleep during daytime periods; however, the circadian alert signal is high at this time, which results in short, fragmented sleep and homeostatic sleep debt. Conversely, when shift workers need to be awake, the circadian alert signal is low, causing sleepiness, long sleep duration in their workplace. Taken together, shift workers may be more vulnerable to experiencing shorter or longer sleep durations that have negative effects on suicidal ideation. Therefore, maintaining an appropriate sleep duration can be a crucial modifiable factor that can reduce the risk of suicidal ideation in shift workers.

In terms of the relationship between long working hours and suicide mortality, Lee et al.'s ${ }^{11}$ study using nationally representative longitudinal data have reported that those working $\geq 45$ hours/week have a higher risk of suicide when compared with individuals working 35-44 hours/week. However, their upper limit of working hours is lower than the result of our study. There are several reasons that account for the difference in working hours that increases the risk of suicide between studies. We included shift workers only, whereas Lee et al. ${ }^{11}$ assessed all employees, including non-shift workers. In addition, the dependent variables are also different between our study (suicidal ideation) and Lee et al. ${ }^{11}$ (suicidal mortality) and we excluded individuals with depressive disorders. A current systematic review study has reported that individuals working 4 hours-on/8 hours-off rosters averaged 1 hour more sleep per night than those working 6 hours-on/ 6 hours-off and 1.3 hours more sleep than those working 8 hours-on/8 hours-off in a shift work system. ${ }^{23}$ Maintaining an appropriate sleep duration is associated with reducing mental health problems, including suicide for shift workers; ${ }^{22}$ therefore, long working hours that result in sleep deficits are additional risk factors in the development of suicidal ideation. Furthermore, long working hours have negative effects on the relationships with family and friends. ${ }^{11}$ Most activities occur during the day; therefore, shift work is more likely to interfere with social obligations and/or family life, which can cause social isolation and family conflict that is associated with suicidal ideation. ${ }^{7,24}$

There were interactions by sex in the association between long working hours and the risk of suicidal ideation. In the current study, the relationship was stronger for men than for women. According to the expectations of cultural gender roles in East Asian cultures, men tend to focus on paid work because their self-identity is often derived from the breadwinner role. ${ }^{25}$ Therefore, male shift worker can be more vulnerable to external social and economic stressors related to the workplace than female employees. ${ }^{26}$ In addition, in the interactions by age groups in the relationship between long working hours and suicidal ideation, the relationship was stronger for those aged $<45$ years than for those aged $\geq 45$ years. Previous studies have reported that mood fluctuation, mean levels of neuroticism decrease as people age. ${ }^{27}$ In addition, increased positivity with age is evident in job attitudes; older workers tend to report higher satisfaction with their job, pay, and supervisor, and higher levels of organizational identification and loyalty. ${ }^{28}$ Accordingly, it can be understandable that younger employees are more vulnerable to suicidal ideation than those aged $\geq 45$ years under long working hours.

The results of this study should be interpreted with caution because of several potential limitations. First, study design was cross-sectional; therefore, inflated associations and reverse causality are possible. Second, self-reported questionnaires were used to identify whether participants experienced suicidal ideation or not, and their working and sleep hours; therefore, our results may be affected by response bias. Third, we measured suicidal ideation alone as an outcome. Even if suicidal ideation is a distinct risk factor of suicide, information on suicidal behavior or actual suicide is more desirable in examining the association between sleep duration, working hours, and suicide. Fourth, this study included only subjective sleep duration as a dependent variable; however, as sleep quality should be used to evaluate restorative sleep. A future study that includes sleep quality-related parameters is required. Fifth, psychosocial job stress, including poor physical working conditions, high job demands, low job control, effort-reward imbalance, inadequate social support, job insecurity, organizational injustice, and discomfort in an organizational climate have been reported as crucial risk factors of suicide. The present study did not include different job stresses as covariates. Sixth, the rate number of individuals who reported "others" when reporting their shift work pattern was high. This suggests that individuals may be misclassified because of incorrect responses. Seventh, this study did not include any multiple person-level characteristics that are associated with adaptability to shift work as covariates. For example, chronotype, which is an individual's innate circadian preference (morning/evening types) can influence an individual's ability to adapt to shift work.

\section{Acknowledgments \\ None.}

\section{Conflicts of Interest}

The authors have no potential conflicts of interest to disclose.

\section{Author Contributions}

Conceptualization: Sun-Young Kim. Data curation: Sun-Young Kim. Formal analysis: Sun-Young Kim. Funding acquisition: Weon-Jeong Lim. Investigation: Sun-Young Kim. Methodology: Sun-Young Kim. Project administration: Weon-Jeong Lim, Soo In Kim. Resources: Sun-Young Kim. Software: Sun-Young Kim. Supervision: Soo In Kim. Validation: Sun-Young Kim. Visualization: Sun-Young Kim. Writing_original draft: Sun-Young Kim. Writing-review \& editing: Sun-Young Kim, Soo In Kim. 


\section{ORCID iDs}

Sun-Young Kim

Soo In Kim

Weon-Jeong Lim

https://orcid.org/0000-0003-3705-9746

https://orcid.org/0000-0001-6909-5034

https://orcid.org/0000-0002-2100-4233

\section{REFERENCES}

1. World Health Organization (WHO). Mental health: suicide prevention. Available at: http://www.who.int/mental_health/suicide-prevention/ en/. Accessed May 29, 2020.

2. Organization for Economic Co-operation and Development (OECD). Suicide rates. Available at: https://www.oecd-ilibrary.org/social-issuesmigration-health/suicide-rates/indicator/english_a82f3459-en. Accessed May 29, 2020.

3. Aleman A, Denys D. Mental health: a road map for suicide research and prevention. Nature 2014;509:421-423.

4. Boivin DB, Boudreau P. Impacts of shift work on sleep and circadian rhythms. Pathol Biol (Paris) 2014;62:292-301.

5. Sixth European survey on working conditions. Available at: https:// www.eurofound.europa.eu/surveys/european-working-conditionssurveys/sixth-european-working-conditions-survey-2015. Accessed September 21, 2020.

6. Cheng P, Drake C. Shift work disorder. Neurol Clin 2019;37:563-577.

7. Kim KK, Lee KR, Suh HS, Ko KD, Hwang IC. Association between shift work and suicidal ideation: data from the Korea National Health and Nutrition Examination Survey (2008-2016). Scand J Work Environ Health 2019;45:458-464.

8. Wright KP, Bogan RK, Wyatt JK. Shift work and the assessment and management of shift work disorder (SWD). Sleep Med Rev 2013;17:4154.

9. Brown JP, Martin D, Nagaria Z, Verceles AC, Jobe SL, Wickwire EM. Mental Health consequences of shift work: an updated review. Curr Psychiatry Rep 2020;22:7.

10. Härmä M, Karhula K, Puttonen S, Ropponen A, Koskinen A, Ojajärvi A, et al. Shift work with and without night work as a risk factor for fatigue and changes in sleep length: a cohort study with linkage to records on daily working hours. J Sleep Res 2019;28:e12658.

11. Lee HE, Kim I, Kim HR, Kawachi I. Association of long working hours with accidents and suicide mortality in Korea. Scand J Work Environ Health 2020;46:480-487.

12. Korea Centers for Disease Control and Prevention (KCDC). About Korea National Health and Nutrition Examination Survey (KNHANES). Available at: https://knhanes.cdc.go.kr/knhanes/eng/index.do. Accessed June 4, 2020.

13. Knutsson A. Shift work and coronary heart disease. Scand J Soc Med Suppl 1989;44:1-36.
14. Hirshkowitz M, Whiton K, Albert SM, Alessi C, Bruni O, DonCarlos L, et al. National Sleep Foundation's updated sleep duration recommendations: final report. Sleep Health 2015;1:233-243.

15. National Legal Information Center. Labor Standard Act. Available at: http://www.law.go.kr/lsInfoP.do?lsiSeq=218277\&efYd=20200526\#0000 Accessed June 4, 2020.

16. Kim SH, Ahn J, Ock M, Shin S, Park J, Luo N, et al. The EQ-5D-5L valuation study in Korea. Qual Life Res 2016;25:1845-1852.

17. Statistics Korea. The Korean Standard Classification of Occupations. Available at: http://kssc. kostat.go.kr/ksscNew_web/ekssc/main/main. do\#. Accessed June 4, 2020.

18. Dolsen MR, Prather AA, Lamers F, Penninx B. Suicidal ideation and suicide attempts: associations with sleep duration, insomnia, and inflammation. Psychol Med 2020:1-10. [Online ahead of print]

19. Gunnell D, Chang SS, Tsai MK, Tsao CK, Wen CP. Sleep and suicide: an analysis of a cohort of 394,000 Taiwanese adults. Soc Psychiatry Psychiatr Epidemiol 2013;48:1457-1465.

20. Littlewood DL, Kyle SD, Carter LA, Peters S, Pratt D, Gooding P. Short sleep duration and poor sleep quality predict next-day suicidal ideation: an ecological momentary assessment study. Psychol Med 2019; 49:403-411.

21. Goldman-Mellor S, Olfson M, Lidon-Moyano C, Schoenbaum M. Mortality following nonfatal opioid and sedative/hypnotic drug overdose. Am J Prev Med 2020;59:59-67.

22. Brown JP, Martin D, Nagaria Z, Verceles AC, Jobe SL, Wickwire EM. Mental health consequences of shift work: an updated review. Curr Psychiatry Rep 2020;22:7.

23. Short MA, Agostini A, Lushington K, Dorrian J. A systematic review of the sleep, sleepiness, and performance implications of limited wake shift work schedules. Scand J Work Environ Health 2015;41:425-440.

24. Van Orden KA, Witte TK, Cukrowicz KC, Braithwaite SR, Selby EA, Joiner TE Jr. The interpersonal theory of suicide. Psychol Rev 2010; 117:575-600.

25. Kang MY, Kwon HJ, Choi KH, Kang CW, Kim H. The relationship between shift work and mental health among electronics workers in South Korea: a cross-sectional study. PLoS One 2017;12:e0188019.

26. Möller-Leimkühler AM. The gender gap in suicide and premature death or: why are men so vulnerable? Eur Arch Psychiatry Clin Neurosci 2003;253:1-8.

27. Ng TWH, Feldman DC. The relationships of age with job attitudes: a meta-analysis. Pers Psychol 2010;63:677-718.

28. Scheibe S, Zacher H. A Lifespan Perspective on Emotion Regulation, Stress, and Well-being in the Workplace. In: Perrewé PL, Rosen CC, Halbesleben JR, Editors. The Role of Emotion and Emotion Regulation in Job Stress and Wellbeing. Bingley: Emerald Group Publishing Limited, 2013, p.163-193. 